Tema: Tratamento Térmico

\title{
ESTUDO DA EVOLUÇÃO MICROESTRUTURAL DE LIGAS DE ALUMÍNIO SUBMETIDAS A TRATAMENTOS TÉRMICOS DE SOLUBILIZAÇÃO COM TEMPOS DISTINTOS DE ENCHARQUE*
}

\section{Resumo}

O presente trabalho estuda as características microestruturais e mecânicas de 3 ligas de alumínio $(1100,3104$ e 8011) na condição laminada a quente e submetidas a um tratamento térmico de solubilização em diferentes tempos de encharque (1, 2 e 3 horas). As ligas em estudo apresentam um percentual de soluto ligeiramente acima do limite máximo de solubilidade nas mesmas. Assim os tratamentos térmicos de solubilização permitiriam controlar a dimensão, dispersão e natureza dos precipitados presentes modificando a condição laminada a quente, sem atingir a completa dissolução dos mesmos. A avaliação microestrutural/ composicional e mecânica foi feita por Microscopia Eletrônica de Varredura (MEV)/ Espectroscopia de Raios-X de Dispersão de Energia (EDS), Difração de Raios-X (DRX) e ensaios de dureza. De acordo com os resultados apresentados para as ligas 1100 e 8011 o tempo para ocorrer à solubilização está entre 1 e 2 horas enquanto que para a liga 3104 está entre os tempos de 2 e 3 horas.

Palavras-chave: Ligas de alumínio; Precipitados; Tratamento térmico de solubilização.

\section{STUDY OF MICROSTRUCTURAL EVOLUTION OF ALUMINIUM ALLOYS SUBJECTED TO SOLUBILIZATION HEAT TREATMENTS WITH DISTINCT HOLDING}

\section{Abstract}

This work studies the microstructural characteristics and mechanical properties of three aluminium alloys $(1100,3104$ e 8011$)$ in hot rolled condition and subjected to subsequent heat treatment of solubilisation. The alloys under study have a solute fraction slightly larger than the maximum solubility limit. So, the solution heat treatments allow the control of the size, nature and dispersion of the precipitates, modifying the hot rolling condition, without complete dissolution of these precipitates. The microstructural/ compositional and mechanical analyses were performed by Scanning Electron Microscopy (SEM)/ Energy Dispersive X-ray Spectroscopy (EDS), X-ray diffraction (XRD) and hardness tests. According to the results for the alloys 1100 and 8011 the time for this solubilization to occur between 1 and 2 hours while for the alloy 3104 is between 2 and 3 hours.

Keywords: Aluminum alloys; Precipitates; Solution heat treatment.

1 M.Sc. em Eng. Metalúrgica, Engenheiro Metalurgista, Doutorando em Ciências dos Materiais, IME, Rio de Janeiro, RJ, Brasil.

2 Engenheiro Metalurgista, trainee, Gerdau, Santa Cruz, RJ, Brasil.

3 Msc., Técnico do Lab. de Microscopia Eletrônica, EEIMVR, UFF, Volta Redonda, RJ, Brasil.

4 D.Sc., Professora Adjunta, EEIMVR-VMT e PPGEM, UFF, Volta Redonda, RJ, Brasil.

5 Engenheira Metalurgista, trainee, Votorantim Metais, Alumínio, SP, Brasil.

* Contribuição técnica ao 69 Congresso Anual da ABM - Internacional e ao 14 ENEMET - Encontro Nacional de Estudantes de Engenharia Metalúrgica, de Materiais e de Minas, 21 a 25 de julho de 2014, São Paulo, SP, Brasil. 


\section{INTRODUÇÃO}

Dentre todos os metais existentes o alumínio é o terceiro em abundância de seu minério na crosta terrestre, e por apresentar uma série de vantagens é o segundo metal mais produzido mundialmente [1], tais vantagens se devem há uma grande gama de propriedade que são resultados da adição de elementos de liga e tratamentos termomecânicos.

O tratamento térmico no seu sentido mais amplo refere-se a qualquer operações de aquecimento e resfriamento que são realizados com a finalidade de mudar as propriedades mecânicas, estrutura metalúrgica, e / ou estado de tensão residual do metal [2].

Dentre os tratamentos térmicos existentes para o alumínio e suas ligas o tratamento térmico de solubilização é utilizado para produzir uma condição estrutural monofásica supersaturada sem precipitados, com maior facilidade para a conformação a frio, ou como condição prévia para o tratamento térmico de envelhecimento. Em casos de ligas onde o teor de soluto presente ultrapassa o limite de solubilidade, esta condição inicial não pode ser alcançada (estrutura monofásica sem precipitados), porém permite-se obter uma matriz supersaturada com uma fração parcial de precipitados. Condição esta que permite a redução na resistência e aumento da ductilidade, porém ainda a contribuição da presença de precipitados de modo a garantir algum controle no crescimento de grão em operações / processamentos posteriores em temperaturas elevadas $[3,4]$.

Um pré-requisito para completa dissolução é a capacidade de aquecer a liga a uma gama de temperatura em que todo o soluto esteja dissolvido, de modo que uma estrutura de fase única seja atingida.

O tratamento térmico de solubilização consiste em aquecer uma liga à uma temperatura suficientemente elevada, no qual é definida pela composição química da liga, durante um tempo suficientemente longo para conseguir uma solução sólida quase homogénea, no qual todo o soluto esteja dissolvido, de modo que uma estrutura de fase única seja atingida [2].

O superaquecimento deve ser evitado, para não ultrapassar a temperatura inicial de fusão eutética, caso isso ocorra, as propriedades, tais como resistência à ruptura, a ductilidade e tenacidade à fratura podem ser degradadas.

No tratamento térmico de solubilização o tempo de encharque, é um parâmetro muito importante, pois é necessário para efetuar um grau satisfatório de solução dos constituintes da fase solúveis não dissolvidas ou precipitadas e para alcançar uma boa homogeneidade da solução sólida antes do tratamento térmico. O tempo de encharque pode variar desde menos de um minuto para folhas finas de alumínio, quanto $20 \mathrm{~h}$, para grandes áreas [2].

O objetivo deste presente trabalho foi o estudo da evolução microestrutural quanto aos precipitados presentes nas ligas de alumínio 1100, 3104 e 8011, submetidas a tratamentos térmicos de solubilização em tempos distintos. De forma a relacionar as mudanças microestruturais com a evolução da propriedade mecânica de dureza obtida com base nos tempos de encharque do tratamento térmico de solubilização realizado.

\footnotetext{
* Contribuição técnica ao 69ำ Congresso Anual da ABM - Internacional e ao 14ํㅡㄹ ENEMET - Encontro Nacional de Estudantes de Engenharia Metalúrgica, de Materiais e de Minas, 21 a 25 de julho de 2014, São Paulo, SP, Brasil.
} 


\section{MATERIAIS E MÉTODOS}

\subsection{Materiais}

Os materiais de estudo foram chapas de ligas de alumínio laminadas a quente de nomenclaturas 1100, 3104 e 8011, com espessuras de 6,00, 10,00 e 6,95 mm, respectivamente, doadas pela Votorantim Metais. Tais chapas de alumínio apresentam as seguintes composições químicas descritas na Tabela 1.

Tabela 1. Composição química das ligas em estudo, em percentual em peso

\begin{tabular}{|c|c|c|c|c|c|c|c|c|c|c|c|}
\hline \multicolumn{10}{|c|}{ Composição química (\%) } \\
\hline Liga & $\mathbf{S i}$ & $\mathbf{F e}$ & $\mathbf{C u}$ & $\mathbf{M n}$ & $\mathbf{M g}$ & $\mathbf{T i}$ & $\mathbf{C r}$ & $\mathbf{N i}$ & $\mathbf{Z n}$ & $\mathbf{A l}$ & Outros \\
\hline $\mathbf{1 1 0 0}$ & 0,14 & 0,52 & 0,096 & 0,009 & 0,001 & 0,012 & 0,005 & 0,005 & 0,005 & 99,20 & 0,007 \\
\hline $\mathbf{3 1 0 4}$ & 0,23 & 0,64 & 0,072 & 0,77 & 0,81 & 0,018 & 0,034 & 0,004 & 0,021 & 97,38 & 0,021 \\
\hline $\mathbf{8 0 1 1}$ & 0,59 & 0,69 & 0,027 & 0,005 & 0,001 & 0,019 & 0,003 & 0,004 & 0,006 & 98,620 & 0,035 \\
\hline
\end{tabular}

\subsection{Métodos}

Amostras de $1 \mathrm{~cm}$ de largura (direção transversal - DT) e $2 \mathrm{~cm}$ (direção de laminação - DL) foram extraídas das distintas chapas através de uma máquina de corte com disco abrasivo de Carbeto de Silicio, sob refrigeração. As amostras extaídas das chapas foram submetidas a um tratamento térmico de solubilização, no qual se procedeu a um aquecimento com temperatura de encharque a $500^{\circ} \mathrm{C}$, durante os tempos de encharque de 1,2 e 3 horas. Após o tempo decorrido as amostras foram resfriadas em água sob agitação.

De modo a conduzir as análises, as amostras foram embutidas a quente de modo a poder-se proceder a preparação metalográfica da seção transversal associada a direção de laminação (DL). As amostras embutidas foram lixadas com lixas de carbeto de silício (granulometria 220 a 4.000 mesh) seguido de polimento com pastas de diamante de $6 \mu \mathrm{m}, 3 \mu \mathrm{m}$ e $1 \mu \mathrm{m}$.

Foi realizado o ensaio de dureza Vickers em um microdurômetro (Fabricante Spectru Instrumental Científico LTDA) com uma carga de $100 \mathrm{~g}(0,1 \mathrm{kgf})$ e tempo de permanência de 18 segundos para as ligas na condição de como recebida (nomeado de $\mathrm{CR}$ ) e nos distintos tempos de tratamento térmico de solubilização (1, 2 e 3 horas, nomeados de TT-1, TT-2 e TT-3, respectivamente). Para cada amostra foram realizadas 10 medidas de microdureza a $1 / 4$ e $1 / 2$ da espessura. Tal procedimento foi realizado para verificar se há variação microestrutural/ mecânica significativa ao longo da espessura dos materiais em estudo.

As caracterizações microestruturais foram realizadas por meio de Microscopia Eletrônica de Varredura (MEV) com intuito de observar a presença de precipitados bem como foram feitas análises de composição química elementar através da sonda de EDS (Espectrômetro de Raios-X de Dispersão de Energia), em pontos que acreditava-se ser os precipitados. Os parâmetros utilizados foram: tensão de aceleração de $12 \mathrm{kV}$ com detector de elétrons secundários (spot size de 500 e distância de trabalho de $11 \mathrm{~mm}$ ).

A análise de difração de Raios-X foi efetuada com o objetivo de identificar as fases presentes nas amostras, no qual foi realizada com um difratômetro com radiação CoK $\alpha$ e goniômetro padrão. Foi aplicada uma tensão de $30 \mathrm{kV}$ e $30 \mathrm{~mA}$ de corrente

\footnotetext{
* Contribuição técnica ao 69 Congresso Anual da ABM - Internacional e ao 14ํㅡㄹ ENEMET - Encontro Nacional de Estudantes de Engenharia Metalúrgica, de Materiais e de Minas, 21 a 25 de julho de 2014, São Paulo, SP, Brasil.
} 
durante as varreduras com acoplamento de $\theta / 2 \theta$ no intervalo de $20^{\circ}$ a $110^{\circ}$ para $2 \theta$, como intervalo de $2 \theta$ e velocidade de $2^{\circ}$ por minuto (equivalente ao tempo de aquisição de 0,6 s por ponto). As fichas JCPDS (Joint Committee on Powder Diffraction Standards) utilizadas nestes trabalhos foram 89-4037 (fase $\alpha$ ), 89-1981 $\left(\mathrm{Al}_{2} \mathrm{Cu}\right.$ ), 65-5188 (AlFe3), 89-7376 (FeSi) e 89-9056 (Si) e 89-9056 (Mg $\left.{ }_{32} \mathrm{Al}_{47} \mathrm{Cu}_{7}\right)$.

\section{RESULTADOS E DISCUSSÃO}

\subsection{Análise Microestrutural e Composicional por MEV/EDS}

As análises microestruturais e composicional por MEV/EDS foram realizadas em regiões a $1 / 4$ e $1 / 2$ da espessura das amostras, mas devido não ter mudanças significativas nas microestruturas e composição química dos precipitados quando se comparado o posicionamento da análise, ou seja, a 1/4 e $1 / 2$ da espessura, serão apenas apresentados os resultados das análises realizadas a $1 / 2$ da espessura, pois estas representam as amostras como um todo.

As Figuras de 1 a 3 apresentam a microestrutura observada através do MEV referente as liga 1100, 3104 e 8011 , respectivamente, observadas a 1/2 espessura em suas distintas condições estudadas (CR, TT-1, TT-2 e TT-3). Os principais precipitados são destacados nas imagens por índices numéricos, os quais são referenciados pela sua composição química avaliada pela sonda de EDS e listados nas Tabelas de 2 a 4.
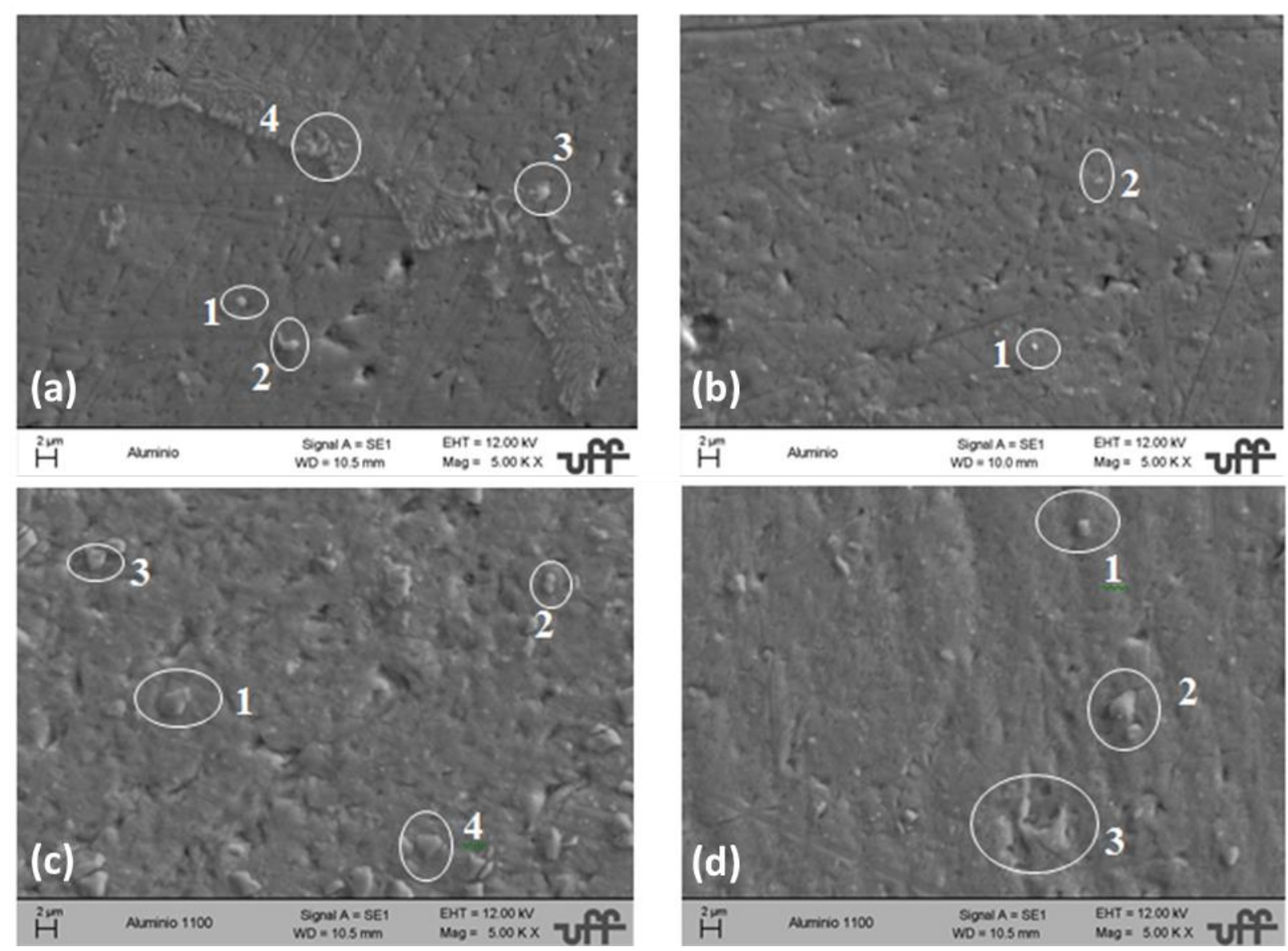

Figura 1. Microestrutura obtida por MEV (Elétrons Secundários) para a região de $1 / 2$ espessura para a liga 1100. (a) condição CR, (b) condição TT-1, (c) condição TT-2 e (d) condição TT-3.

* Contribuição técnica ao 69 Congresso Anual da ABM - Internacional e ao 14ํㅡN ENET - Encontro Nacional de Estudantes de Engenharia Metalúrgica, de Materiais e de Minas, 21 a 25 de julho de 2014, São Paulo, SP, Brasil. 
Tabela 2. Composição química da liga 1100 a 1/2 espessura, percentual em peso, realizado por EDS em diferentes pontos indicados na Figura 1

\begin{tabular}{|c|c|c|c|c|c|c|c|c|c|c|c|c|c|c|}
\hline \multirow{3}{*}{\multicolumn{2}{|c|}{$\begin{array}{c}\text { Amostra } \\
\text { Condiçao } \\
\text { Ponto }\end{array}$}} & \multicolumn{13}{|c|}{$1100-1 / 2$ Espessura } \\
\hline & & \multicolumn{4}{|c|}{ CR (a) } & \multicolumn{2}{|c|}{ Tा-1 (b) } & \multicolumn{4}{|c|}{$\pi T-2(c)$} & \multicolumn{3}{|c|}{$T T-3$ (d) } \\
\hline & & 1 & 2 & 3 & 4 & 1 & 2 & 1 & 2 & 3 & 4 & 1 & 2 & 3 \\
\hline \multirow{5}{*}{$\begin{array}{c}\text { Elemento } \\
\text { (\%) em } \\
\text { peso }\end{array}$} & Si & - & 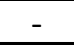 & - & - & - & 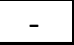 & 38,7 & 6,9 & 20,1 & 30,0 & 13,5 & 29,8 & 8,1 \\
\hline & $\mathrm{Fe}$ & 5,0 & 7,3 & 9,1 & 11,7 & 3,9 & 3,8 & 0,7 & 1,0 & 1,9 & 1,2 & 0,8 & 0,8 & 1,9 \\
\hline & $\mathrm{Cu}$ & 1,5 & 1,7 & 2,1 & 1,8 & 1,4 & 1,4 & - & - & 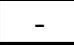 & - & - & - & - \\
\hline & Mg & - & - & - & - & - & - & - & - & - & - & 0,4 & 0,3 & 0,4 \\
\hline & Outros • & 0,7 & 0,7 & 0,7 & 0,6 & 0,8 & 0,7 & 7,5 & 7,2 & 8,9 & 5,8 & 2,4 & 11,7 & 1,6 \\
\hline
\end{tabular}
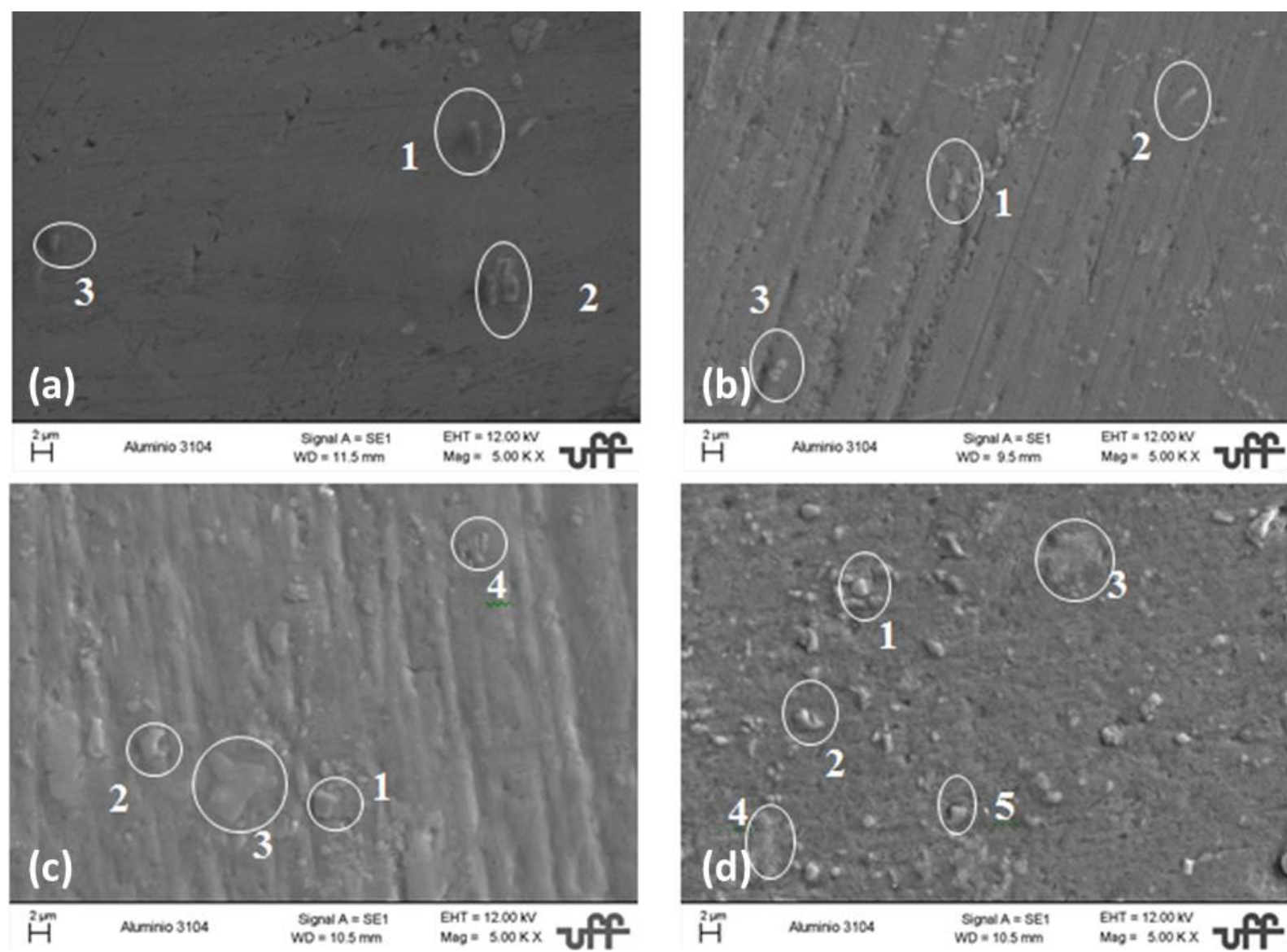

Figura 2. Microestrutura obtida por MEV (Elétrons Secundários) para a região de $1 / 2$ espessura para a liga 3104. (a) condição CR, (b) condição TT-1, (c) condição TT-2 e (d) condição TT-3.

Tabela 3. Composição química da liga 3104 a $1 / 2$ da espessura, percentual em peso, realizado por EDS em diferentes pontos indicados na Figura 2

\begin{tabular}{|c|c|c|c|c|c|c|c|c|c|c|c|c|c|c|c|c|}
\hline \multirow{3}{*}{\multicolumn{2}{|c|}{$\begin{array}{c}\text { Amostra } \\
\text { Condiçao } \\
\text { Ponto }\end{array}$}} & \multicolumn{15}{|c|}{$3104-1 / 2$ Espessura } \\
\hline & & \multicolumn{3}{|c|}{ CR (a) } & \multicolumn{3}{|c|}{$\pi-1$ (b) } & \multicolumn{4}{|c|}{$\pi T-2(c)$} & \multicolumn{5}{|c|}{$\pi T-3(d)$} \\
\hline & & 1 & 2 & 3 & 1 & 2 & 3 & 1 & 2 & 3 & 4 & 1 & 2 & 3 & 4 & 5 \\
\hline \multirow{6}{*}{ Elemento } & Si & 4,1 & 3,9 & 3,2 & 2,6 & 8,5 & 2,0 & 38,7 & 6,9 & 20,1 & 26,0 & 12,3 & 18,1 & 2,0 & 0,7 & 2,9 \\
\hline & $\mathrm{Fe}$ & 17,5 & 14,5 & 13,4 & 17,7 & 15,9 & 9,8 & 0,7 & 1,0 & 1,9 & 1,5 & - & - & 6,2 & 3,3 & 6,5 \\
\hline & $\mathrm{Cu}$ & 0,5 & 0,4 & 0,3 & 0,2 & 0,4 & 0,5 & 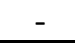 & - & - & - & - & - & - & - & - \\
\hline & $\mathrm{Mn}$ & 8,0 & 7,9 & 6,5 & 3,4 & 5,1 & 3,5 & - & - & - & - & - & - & 3,1 & 1,8 & 3,2 \\
\hline & $\mathrm{Mg}$ & 0,5 & 0,7 & 0,8 & 0,4 & 0,5 & 0,8 & - & - & - & - & 0,8 & 0,8 & 0,9 & 0,9 & 0,8 \\
\hline & Outros $\bullet$ & 23,1 & 32,0 & 34,7 & 5,4 & 8,9 & 5,4 & 7,5 & 7,2 & 8,9 & 5,8 & 10,5 & 6,2 & 3,1 & 3,2 & 2,6 \\
\hline
\end{tabular}

\footnotetext{
* Contribuição técnica ao 69 Congresso Anual da ABM - Internacional e ao 14ํㅡㄹ ENEMET - Encontro Nacional de Estudantes de Engenharia Metalúrgica, de Materiais e de Minas, 21 a 25 de julho de 2014, São Paulo, SP, Brasil.
} 

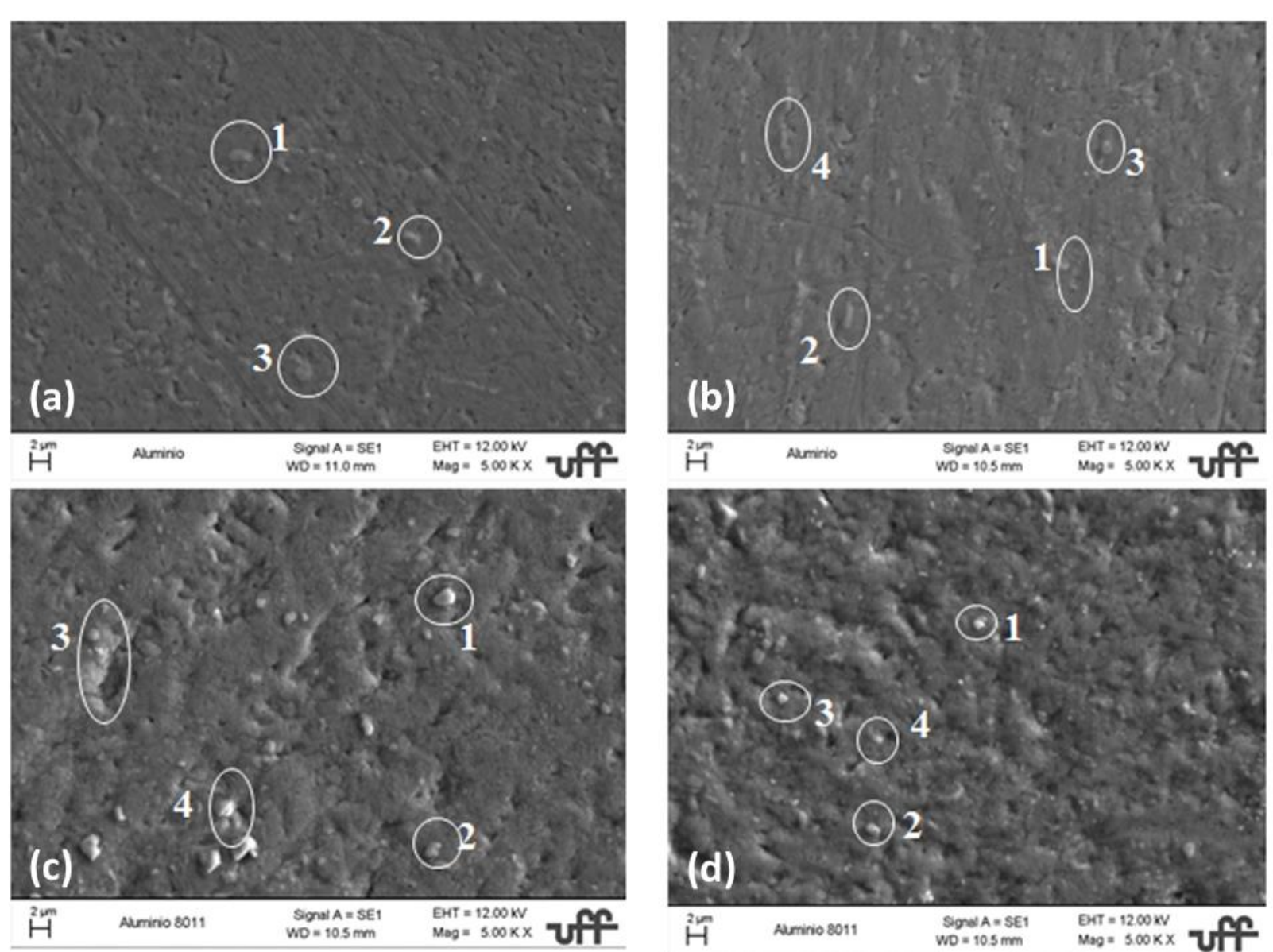

Figura 3. Microestrutura obtida por MEV (Elétrons Secundários) para a região de $1 / 2$ da espessura para a liga 8011. (a) condição CR, (b) condição TT-1, (c) condição TT-2 e (d) condição TT-3.

Tabela 4. Composição química da liga 8011 a $1 / 2$ da espessura, percentual em peso, realizado por EDS em diferentes pontos indicados na Figura 3

\begin{tabular}{|c|c|c|c|c|c|c|c|c|c|c|c|c|c|c|c|c|}
\hline \multirow{3}{*}{\multicolumn{2}{|c|}{$\begin{array}{c}\text { Amostra } \\
\text { Condiçao } \\
\text { Ponto }\end{array}$}} & \multicolumn{15}{|c|}{8011 - 1/2 Espessura } \\
\hline & & \multicolumn{3}{|c|}{ CR (a) } & \multicolumn{4}{|c|}{ TT-1 (b) } & \multicolumn{4}{|c|}{$\pi T-2(c)$} & \multicolumn{4}{|c|}{$\pi-3(d)$} \\
\hline & & 1 & 2 & 3 & 1 & 2 & 3 & 4 & 1 & 2 & 3 & 4 & 1 & 2 & 3 & 4 \\
\hline \multirow{3}{*}{ Elemento } & Si & 8,5 & 4,1 & 1,7 & 5,5 & 0,5 & 1,3 & 3,1 & 47,6 & 22,6 & 4,6 & 2,1 & 10,8 & 13,8 & 3,3 & 13,9 \\
\hline & $\mathrm{Fe}$ & 3,2 & 2,4 & 5,6 & 12,1 & 2,1 & 4,7 & 5,4 & - & - & 8,6 & 4,5 & - & - & 10,2 & 6,6 \\
\hline & Outros & 11,6 & 17,7 & 13,2 & 7,8 & 11,7 & 12,8 & 13,5 & 16,1 & 9,0 & 9,5 & 6,3 & 3,0 & 6,4 & 9,0 & 6,6 \\
\hline
\end{tabular}

Durante a análise da composição química por EDS observou-se a presença de outros elementos químicos (indicados por 'Outros' nas Tabelas 2 , 3 e 4), onde se faziam presentes $\mathrm{O}$ e metais (como o próprio $\mathrm{Al}$ ), possivelmente devido à presença de óxidos do material oriundos do processo de elaboração das ligas.

Fazendo-se uma comparação entre a morfologia dos precipitados encontrados para as ligas 1100, 3104 e 8011 (Figuras 1 a 3), nas distintas condições de análise (condição de CR, TT-1, TT-2 e TT-3) observa-se que estes precipitados são de formatos semelhantes. Para as condições de TT-1 e TT-2 nota-se um aumento dos precipitados em todas as ligas em estudo. Esse aumento de dimensão dos precipitados continuou somente para a liga 3104 para o tempo de 3 horas do tratamento térmico de solubilização (Figura 2), no qual se tornaram mais evidentes e dispersos tanto na forma esferoidal quanto na forma de agulhas.

\footnotetext{
* Contribuição técnica ao 69 Congresso Anual da ABM - Internacional e ao 14ํㅡㄹ ENEMET - Encontro Nacional de Estudantes de Engenharia Metalúrgica, de Materiais e de Minas, 21 a 25 de julho de 2014, São Paulo, SP, Brasil.
} 


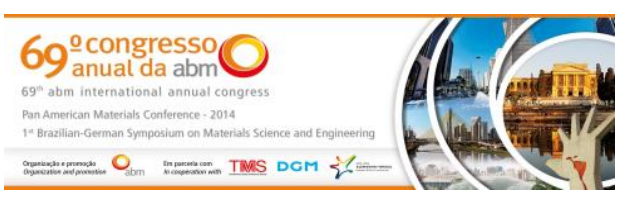

Para as ligas 1100 (Figuras 1) e 8011 (Figura 3) percebe-se que os precipitados tornaram-se mais evidentes e homogêneos na condição TT-2 (Figuras 1c e 3c). Para a condição de TT-3 (Figuras 1d e 3d) verifica-se uma diminuição do número de precipitados provavelmente devido o tempo de solubilização das ligas que deve estar entre duas e três horas. Dessa forma isto indica indício de um provável evento inicial de coalescimento de precipitados nas primeiras horas de tratamento (1 e $2 \mathrm{~h}$ para as ligas 1100 e 3104 , e $1 \mathrm{~h}$ para a liga 8011) e dissolução dos mesmos nos tempos mais longos de tratamento ( $3 \mathrm{~h}$ para as ligas 1100 e 3104, e 2 e $3 \mathrm{~h}$ para a liga 8011).

Com base na composição química das ligas fornecidas pela Votorantim Metais (Tabela 1) e nos resultados obtidos da composição química elementar via análise de EDS (Tabelas de 2 a 4) em diferentes pontos associados a precipitados presentes nas amostras em estudo na região $1 / 2$ espessura (Figuras de 1 a 3 ) nas condições CR, TT-1, TT-2 e TT-3, possivelmente ocorreu uma migração dos átomos do elemento de liga dos precipitados para a matriz. Evidencia esta que pode ser também confirmada pela mudança da morfologia dos precipitados presentes, em comparação com as amostras referentes às condições CR, TT-1, TT-2 e TT-3 (Figuras de 1 a 3 ).

\subsection{Evolução da Dureza}

A Figura 4 faz um comparativo dos resultados dos valores médios e desvios padrão (demonstrado na forma de barra de erros) da dureza Vickers das amostras das ligas em estudo, em relação a sua região de medição, ou seja, 1/4 e 1/2 da espessura, nas condições CR, TT-1, TT-2 e TT-3.
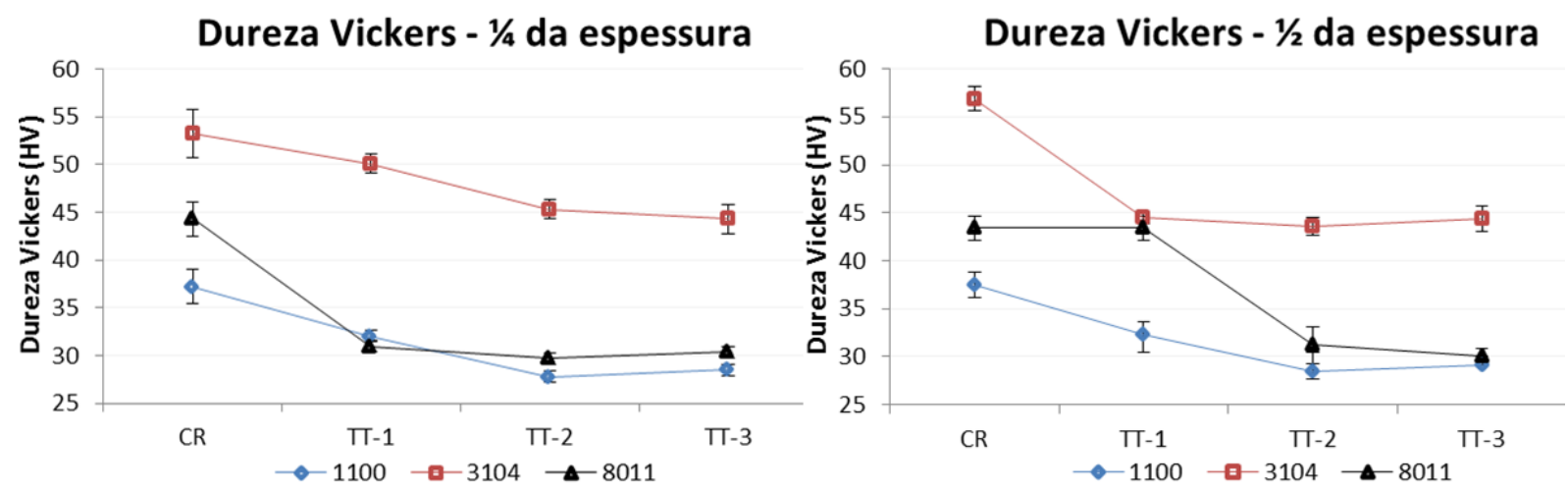

Figura 4. Evolução da dureza Vickers para as ligas em estudo nas condições CR, TT-1, TT-2 e TT-3.

De acordo com a Figura 4, pode-se observar que a dureza entre as regiões de 1/4 e $1 / 2$ espessura de uma mesma amostra, de uma determinada liga, são muito próximas, e com isso, pode-se inferir que há homogeneidade microestrutural entre as regiões analisadas (1/4 e $1 / 2$ da espessura).

Quando se compara as ligas em estudo, observa-se que a liga que possui menor dureza é a liga 1100 e a de maior dureza é a 3104, colocando-se numa condição intermediária a liga 8011. Tal comportamento se deve a composição química de cada liga em estudo, ou seja, a liga 1100 é considerada uma liga comercialmente pura, tendo assim uma menor dureza, e a liga 3104 uma maior dureza devido aos elementos de liga.

* Contribuição técnica ao 69 Congresso Anual da ABM - Internacional e ao 14ํㅡN Nacional de Estudantes de Engenharia Metalúrgica, de Materiais e de Minas, 21 a 25 de julho de 2014, São Paulo, SP, Brasil. 


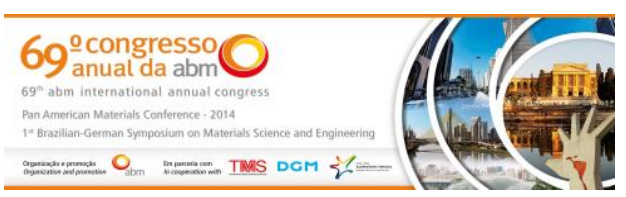

De acordo com a Figura 4, pode-se verificar um decréscimo nos valores da dureza para todas as ligas em estudo com a realização do tratamento térmico de solubilização comparada a condição de CR. Comparando os valores de dureza Vickers obtidos tanto na condição de CR quanto na de TT-1, TT-2 e TT-3, das ligas em estudo, verificou que os valores de dureza mantiveram-se mais altos para a liga 3104, enquanto ocorreu uma aproximação dos valores da liga 8011 (que era de dureza intermediária) com os valores da liga 1100 (que era de menor dureza). Observa-se que para as ligas 1100 e 3104 que as grandes mudanças na dureza (decréscimo da mesma) ocorrem até a condição de TT-2, enquanto que os valores de dureza apresentados para a condição TT-3 são muito próximos a da condição TT-2. No entanto, para a liga 8011 as grandes mudanças na dureza (decréscimo da mesma) ocorrem até a condição TT-1 e mantêm-se próximos para as condições TT-2 e TT-3. Tais resultados das condições TT-1,TT-2 e TT-3 evidenciam em comparação com os resultados da condição $\mathrm{CR}$, a possível incorporação de soluto à matriz pela dissolução de alguns dos precipitados devido à redução da dureza na condição após o tratamento térmico de solubilização, revelando a eficiência do tratamento no seu propósito inicial de produzir uma microestrutura diferenciada daquela da condição de CR. Porém, esta modificação também pode estar associada ao crescimento de grão promovido pela temperatura e tempo [5] de permanência na condição de encharque do tratamento térmico de solubilização.

As adições de elementos como o $\mathrm{Si}, \mathrm{Fe}, \mathrm{Mn}$, Cu aumentam a dureza em ligas de alumínio, tanto por solução sólida (menos pronunciada) como por precipitação (mais significativo em função da natureza química e dimensão dos precipitados), o que justifica os resultados apresentados e sua respectiva [6].

\subsection{Análise de Fases por DRX}

Para uma melhor visualização e análise dos dados obtidos através da difração de Raios-X, elaborou-se gráficos para cada liga estudada (Figuras 5, 6 e 7) onde se sobrepôs os dados das quatro condições analisadas para cada liga. Os valores apresentados nas Figuras 5, 6 e 7 representam os valores das intensidades relativas (em \%), ou seja, o valor demonstrado é a porcentagem em relação à maior intensidade obtida durante o ensaio daquela condição. Os picos associados aos planos de precipitados identificados nestas ligas estão assinalados nas Figuras 5 a 7 por diferentes símbolos, no qual são: $\mathrm{Mg}_{32} \mathrm{Al}_{47} \mathrm{Cu}_{7}(+), \mathrm{Al}_{2} \mathrm{Cu}\left({ }^{*}\right), \mathrm{AlFe}_{3}(\#), \mathrm{Si}(\&) \mathrm{e}$ FeSi (\$).

* Contribuição técnica ao 69ํ Congresso Anual da ABM - Internacional e ao 14ํㅡㄹ ENEMET - Encontro Nacional de Estudantes de Engenharia Metalúrgica, de Materiais e de Minas, 21 a 25 de julho de 2014, São Paulo, SP, Brasil. 

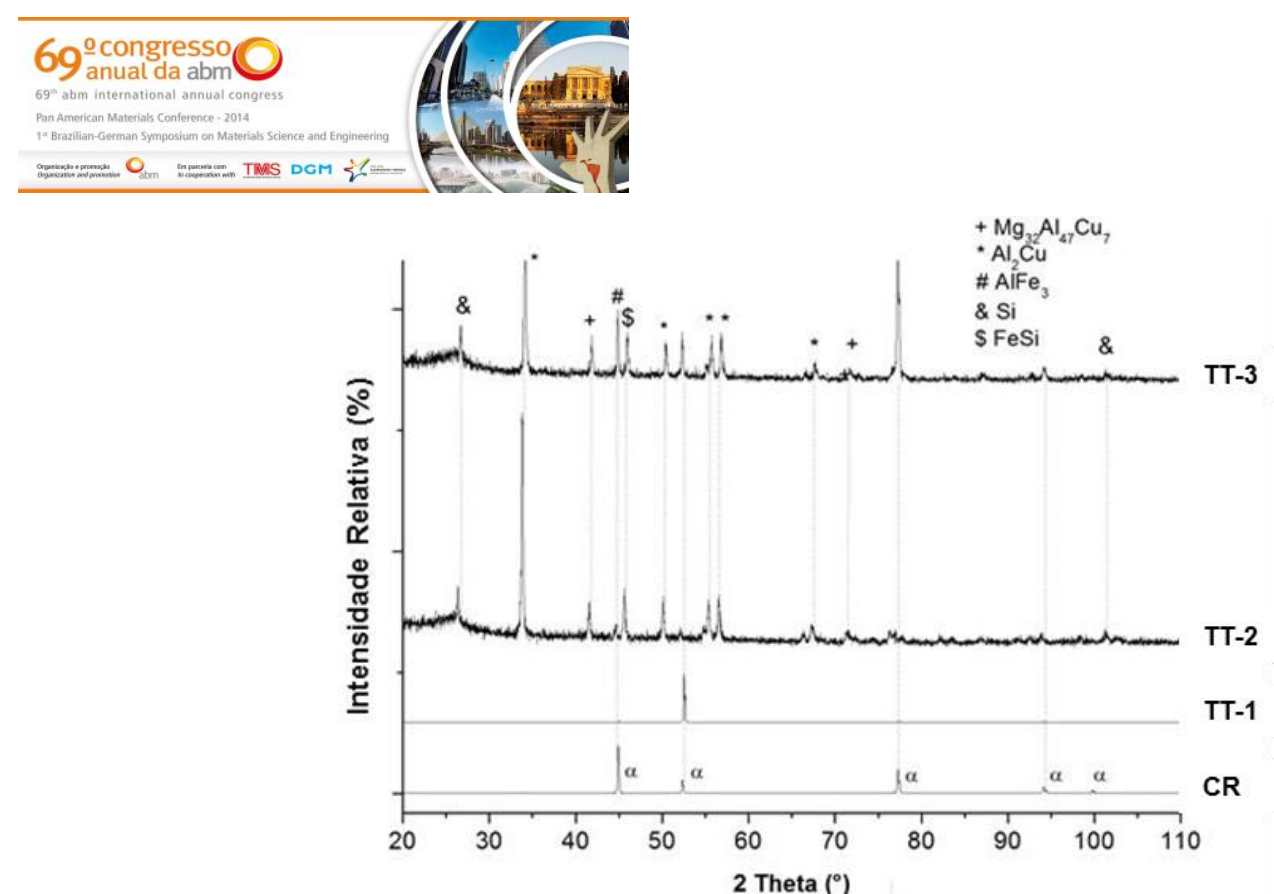

Figura 5. Resultados das análises de difração de Raios-X para a liga 1100, nas condições de CR, TT-1,TT-2 e TT-3.

De acordo com a Figura 5, que representa os dados da análise de DRX para a liga 1100 , pode-se observar a presença da fase $\alpha$ associada à matriz rica em alumínio, através dos seus picos de difração dos planos desta fase, desde a amostra CR à TT-3. Como pode ser observado na Figura 5 nota-se um aumento significativo na intensidade referente os planos dos precipitados da condição TT-1 para a TT-2, dando indícios de que o tempo ideal para atingir a estabilização das fases está entre 1 e 2 horas, no qual este resultado corrobora com as análises do MEV/ EDS (Figura 1 e Tabela 2) e resultados de dureza Vickers (Figura 4).

Os precipitados de $\mathrm{Al}_{2} \mathrm{Cu}$ e $\mathrm{Mg}_{32} \mathrm{Al}_{47} \mathrm{Cu}_{7}$ são os que aparentemente aparecem em maior proporção, com aumento do tempo do tratamento térmico de solubilização, tal fato corrobora com a composição química da liga 1100 (Tabela 1) e contradiz os resultados das análises EDS (Tabelas 2) no qual, indicam que com aumento do tempo de encharque no tratamento térmico de solubilização, ocorre um decréscimo dos percentuais $\mathrm{Cu}$ e incremento dos teores de Si e/ou Fe nos precipitados. Este fato é suportado visualmente pelas modificações dimensionais e morfológicas observadas nas análises no MEV com auxílio do detector de elétrons secundários (Figura 1), que reafirma as possibilidade do crescimento/ coalescência dos precipitados com o tratamento térmico de solubilização, em conjunto com a dissolução de outros precipitados menos estáveis pela homogeneização composicional da matriz.

* Contribuição técnica ao 69ํ Congresso Anual da ABM - Internacional e ao 14ํㅡㄹ ENEMET - Encontro Nacional de Estudantes de Engenharia Metalúrgica, de Materiais e de Minas, 21 a 25 de julho de 2014, São Paulo, SP, Brasil. 

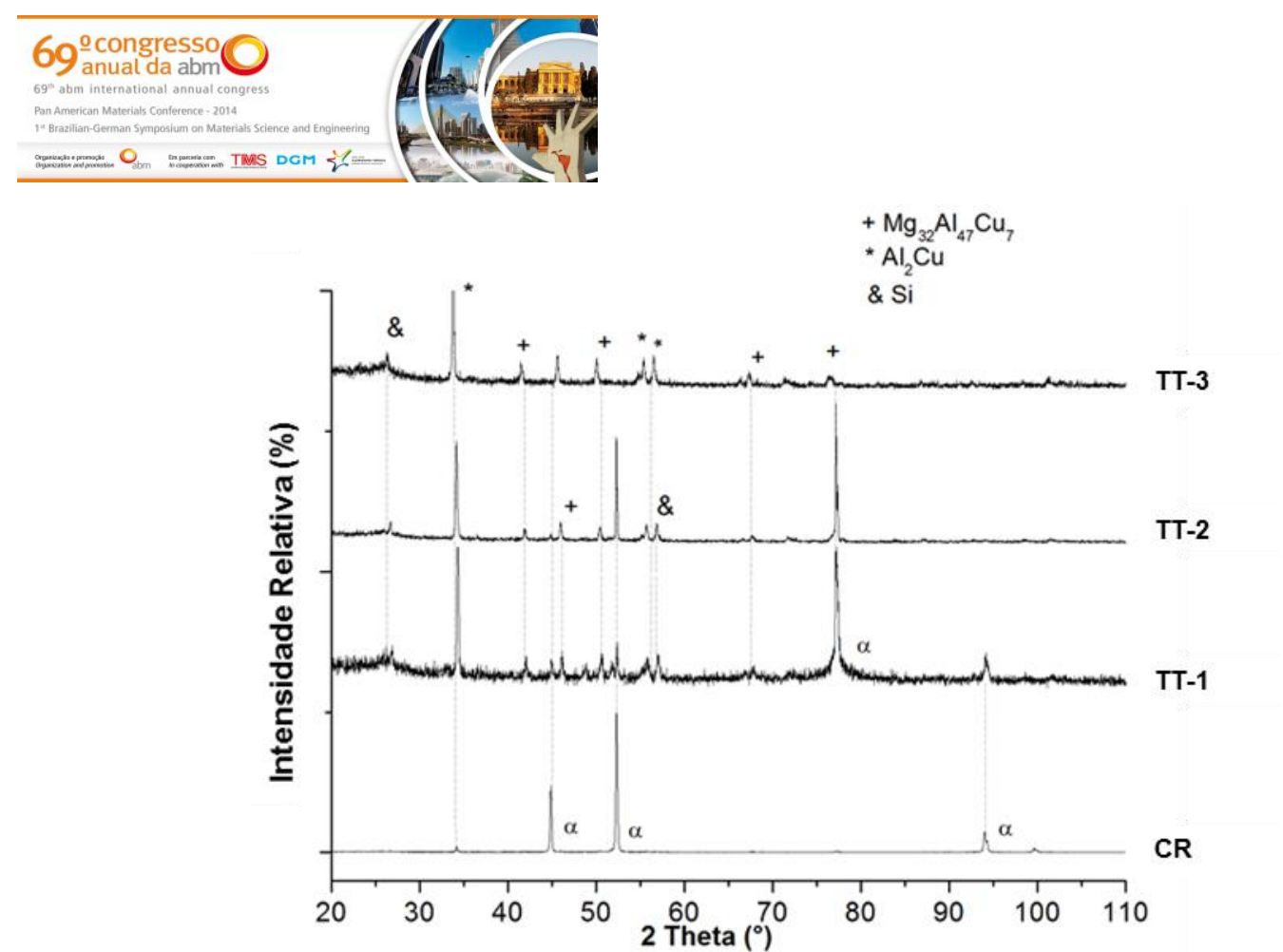

Figura 6. Resultados das análises de difração de Raio-X para a liga 3104, nas condições de CR, TT-1,TT-2 e TT-3.

De acordo com a Figura 6, que representa os dados da análise de DRX para a liga 3104, pode-se observar a presença da fase $\alpha$ associada à matriz rica em alumínio, através dos seus picos de difração dos planos desta fase, desde a amostra CR à TT-3. Como pode ser observado na Figura 6 nota-se um aumento significativo na intensidade referente os planos dos precipitados da condição TT-2 para a TT-3, dando indícios de que o tempo ideal para atingir a estabilização das fases está entre 2 e 3 horas, no qual este resultado corrobora com as análises do MEV / EDS (Figura 2 e Tabela 3) e resultados de dureza Vickers (Figura 4).

Os precipitados de $\mathrm{Al}_{2} \mathrm{Cu}$ e $\mathrm{Mg}_{32} \mathrm{Al}_{47} \mathrm{Cu}_{7}$ são os que aparentemente aparecem em maior proporção, com aumento do tempo do tratamento térmico de solubilização, tal fato corrobora com a composição química da liga 3104 (Tabela 1) e contradiz os resultados das análises EDS (Tabela 3 ) no qual, indicam que com aumento do tempo de encharque no tratamento térmico de solubilização, ocorre um decréscimo dos percentuais $\mathrm{Cu}$ e incremento dos teores de Si e/ou Fe nos precipitados. Este fato é suportado visualmente pelas modificações dimensionais e morfológicas observadas nas análises no MEV com auxílio do detector de elétrons secundários (Figura 2), que reafirma a possibilidade do crescimento / coalescência dos precipitados com o tratamento térmico de solubilização, em conjunto com a dissolução de outros precipitados menos estáveis pela homogeneização composicional da matriz.

* Contribuição técnica ao 69 Congresso Anual da ABM - Internacional e ao 14 ENEMET - Encontro Nacional de Estudantes de Engenharia Metalúrgica, de Materiais e de Minas, 21 a 25 de julho de 2014, São Paulo, SP, Brasil. 

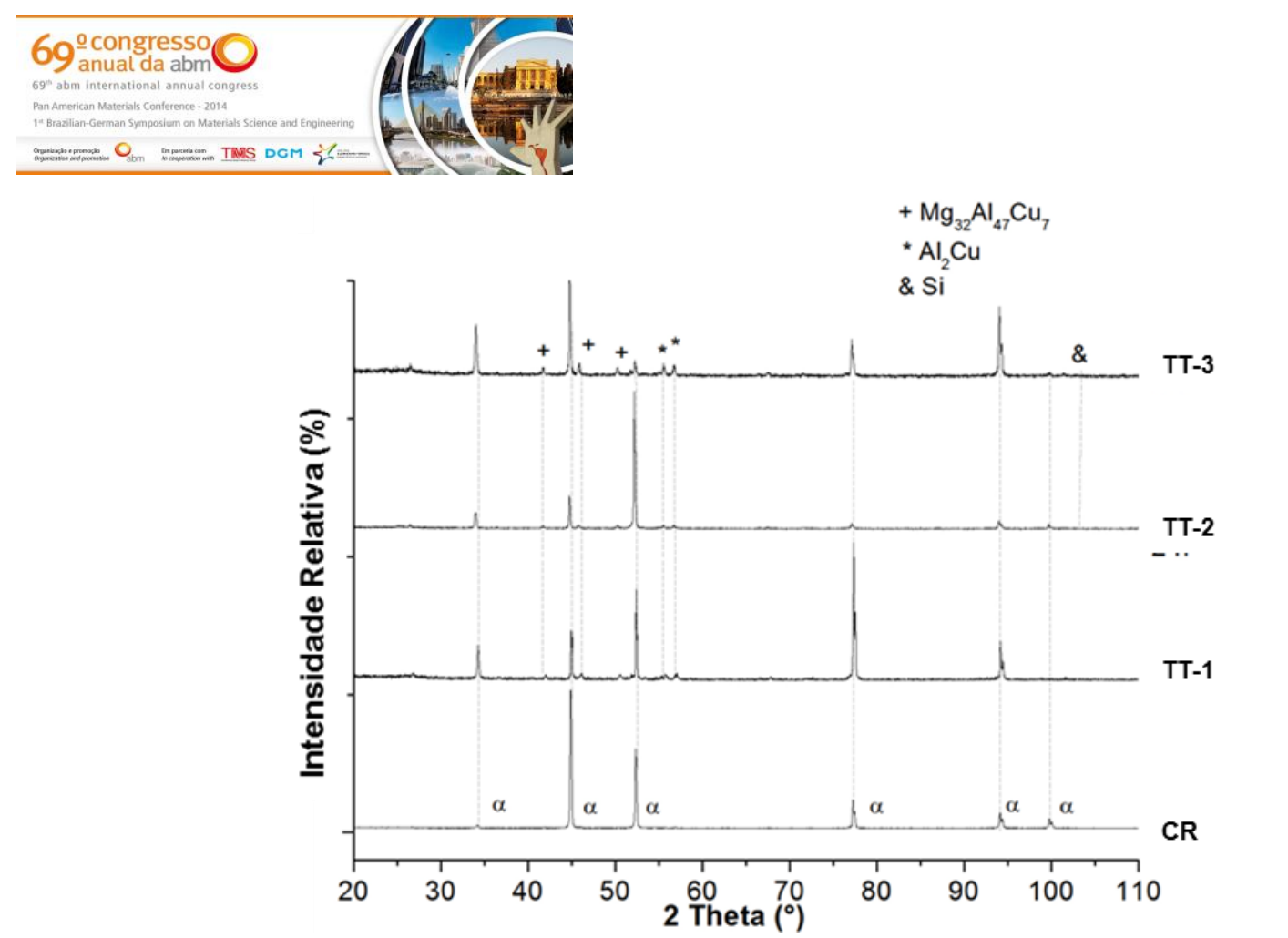

Figura 7. Resultados das análises de difração de Raios-X para a liga 8011 nas condições de CR, TT-1,TT-2 e TT-3.

De acordo com a Figura 7, que representa os dados da análise de DRX para a liga 8011, pode-se observar, assim como as outras ligas, a presença da fase $\alpha$ associada à matriz rica em alumínio, através dos seus picos de difração dos planos desta fase, desde a amostra CR à TT-3. Como pode ser observado na Figura 7 nota-se um aumento significativo na intensidade referente os planos dos precipitados da condição TT-1 para a TT-2, dando indícios de que o tempo ideal para atingir a estabilização das fases está entre 1 e 2 horas, no qual este resultado corrobora com as análises do MEV / EDS (Figura 3 e Tabela 4) e resultados de dureza Vickers (Figura 4).

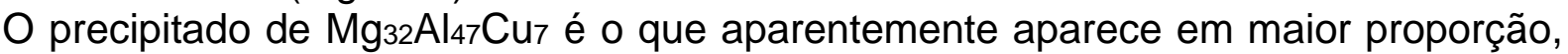
com aumento do tempo do tratamento térmico de solubilização, tal fato corrobora com evidências dos elementos $\mathrm{Mg}$ e Cu detectados na composição química da liga 8011 (Tabela 1), e contradiz os resultados das análises EDS (Tabela 4) onde foram constatadas evidências de precipitados associados a Fe e Si e não relativos ao $\mathrm{Mg}$ e Cu não ocorrendo também à formação de precipitados com o elemento $\mathrm{Fe}$.

Comparando os dados obtidos da análise de difração de Raios-X (Figuras 5, 6 e 7) com os dados de composição química obtidas por EDS (Tabelas de 2 a 4), pode-se constatar nos dados, que o principal elemento para a formação de precipitados é o Cu e o Mg para as três ligas em estudo. Já para a liga 1100 ocorre a formação de compostos que contém o elemento $\mathrm{Fe}$, o fato de não aparecer na análise de EDS não significa a não ocorrência do mesmo, isto pode estar associado a sua dimensão e/ou fração volumétrica estar abaixo do limite de detecção pela análise de DRX. Ou seja, os precipitados associados ao $\mathrm{Si}$ e/ou $\mathrm{Fe}$ apesar de terem uma maior dimensão podem ter uma baixa difratabilidade. Enquanto os precipitados associadas ao $\mathrm{Cu}$ e/ou $\mathrm{Mg}$ apesar de terem um menor dimensão podem ter uma maior difratabilidade.

* Contribuição técnica ao 69ํ Congresso Anual da ABM - Internacional e ao 14ํㅡㄹ ENEMET - Encontro Nacional de Estudantes de Engenharia Metalúrgica, de Materiais e de Minas, 21 a 25 de julho de 2014, São Paulo, SP, Brasil. 


\section{CONCLUSÕES}

Com base nas análises realizadas por ensaios de dureza Vickers, MEV/ EDS e DRX das amostras das ligas 1100, 3104 e 8011 na condição de CR, TT-1, TT-2 e TT-3, pode-se concluir que, o tratamento térmico de solubilização, foi eficaz no que diz respeito a:

- ter resultado em precipitados esferoidais com uma maior dispersão;

- decréscimo da quantidade de precipitados na forma de agulha;

- decréscimo da dureza das amostras pela dissolução e/ou redução dimensional dos precipitados prévios e incorporação de soluto na solução sólida da matriz.

Dessa forma, conclui-se que para as ligas 1100 e 8011 o tempo para ocorrer à solubilização esta entre 1 e 2 horas, enquanto que para a liga 3104 esta entre os tempos de 2 e 3 horas.

\section{Agradecimentos}

Os autores agradecem à Votorantim Metais pelo fornecimento de materiais para execução deste trabalho, a CAPES pela bolsa de mestrado (S. B. Diniz) e ao Laboratório Multiusuário de Microscopia Eletrônica (LMME) da Universidade Federal Fluminense pela utilização do Microscópio Eletrônico de Varredura e equipamentos de preparação e análise de amostras.

\section{REFERÊNCIAS}

1 ABAL. Fundamentos e aplicações do alumínio. São Paulo: Associação Brasileira do Alumínio (ABAL); 2004.

2 ASM Handook, Heat Treating. ASM International, volume 4, 1991.

3 Nieh TG, Wadswoth J, Shelby OD. Superplasticity in metals and ceramics. Nova lorque: Cambridge University Press; 2005.

$4 \mathrm{Xu}$ C, Furukawa M, Horita Z, Langdon TG. Using ECAP to achieve grain refinement, precipitate fragmentation and high strain rate superplasticity in a spraycast aluminum alloy. Acta Materialia, 2003;51:6139-49.

5 Porter DA, Easterling K. Phase transformations in metals and alloys. Nova Iorque: Editora Chapman and Hall; 1992.

6 ASM Handook. Properties and selection: nonferrous alloys and special-purpose materials. Volume 2. ASM International; 1990.

* Contribuição técnica ao 69 Congresso Anual da ABM - Internacional e ao 14ํㅡN Nacional de Estudantes de Engenharia Metalúrgica, de Materiais e de Minas, 21 a 25 de julho de 2014, São Paulo, SP, Brasil. 